Review

\title{
Porcine pancreatic $\alpha$-amylase inhibition by the kidney bean (Phaseolus vulgaris) inhibitor ( $\alpha$-AI1) and structural changes in the $\alpha$-amylase inhibitor complex
}

\author{
Marius Santimone ${ }^{\mathrm{a}}$, Roger Koukiekolo ${ }^{\mathrm{b}}$, Yann Moreau ${ }^{\mathrm{c}}$, Véronique Le Berre ${ }^{\mathrm{d}}$, \\ Pierre Rougée, Guy Marchis-Mouren $^{\mathrm{a}}$, Véronique Desseaux ${ }^{\mathrm{a}, *}$ \\ ${ }^{a}$ Institut Méditerranéen de Recherche en Nutrition (IMRN case 342), UMR INRA 1111, Faculté des Sciences et Techniques de St Jérôme, \\ Université d'Aix-Marseille, Av. Esc. Normandie-Niemen, 13397 Marseilles cedex 20, France \\ ${ }^{\mathrm{b}}$ Section of Molecular and Cellular Biology University of California, One Shields Avenue, Davis, CA 95616, USA \\ ${ }^{\mathrm{c}}$ Institut de Recherche pour le Développement, UR081 Gamet clo CEMAGREF, Montpellier, France \\ ${ }^{\mathrm{d}}$ INSA/DGBA Plateforme Transcriptome Biopuces, 135 Av. de Rangueil 31077, Toulouse, France \\ ${ }^{\mathrm{e}}$ Surface Cellulaire et Signalisation chez les Végétaux UMR-CNRS 5546, 205, Pôle de Biotechnologie Végétale, \\ ch. de Borde-Rouge 31326 Castanet, Tolosan, France
}

Received 3 June 2003; accepted 3 November 2003

\begin{abstract}
Porcine pancreatic $\alpha$-amylase (PPA) is inhibited by the red kidney bean (Phaseolus vulgaris) inhibitor $\alpha$-AI1 [Eur. J. Biochem. 265 (1999) 20]. Inhibition kinetics were carried out using DP 4900-amylose and maltopentaose as substrate. As shown by graphical and statistical analysis of the kinetic data, the inhibitory mode is of the mixed noncompetitive type whatever the substrate thus involving the EI, EI2, ESI and ESI2 complexes. This contrast with the E2I complex obtained in the crystal and with biophysical studies. Such difference very likely depends on the $[\mathrm{I}] /[\mathrm{E}]$ ratio. At low ratio, the E2I complex is favoured; at high ratio the EI, ESI and EI2 complexes are formed. The inhibition model also differs from those previously proposed for acarbose [Eur. J. Biochem. 241 (1996) 787 and Eur. J. Biochem. 252 (1998) 100]. In particular, with $\alpha$-AI1, the inhibition takes place only when PPA and $\alpha-\mathrm{AI}$ are preincubated together before adding the substrate. This indicates that the abortive PPA- $\alpha$ AI1 complex is formed during the preincubation period. One additional carbohydrate binding site is also demonstrated yielding the ESI complex. Also, a second protein binding site is found in EI2 and ESI2 abortive complexes. Conformational changes undergone by PPA upon $\alpha$-AI1 binding are shown by higher sensitivity to subtilisin attack. From X-ray analysis of the $\alpha$-AI1-PPA complex (E2I), the major interaction occurs with two hairpin loops L1 (residues 29-46) and L2 (residues 171-189) of $\alpha$-AI1 protruding into the V-shaped active site of PPA. The hydrolysis of $\alpha$-AI1 that accounts for the inhibitory activity is reported.
\end{abstract}

(C) 2003 Elsevier B.V. All rights reserved.

Keywords: Enzyme kinetics; $\alpha$-Amylase; Amylose; Maltopentaose; Phaseolus vulgaris; Inhibition

\section{Introduction}

The common bean (Phaseolus vulgaris) contains a family of plant defence proteins that includes phytohemagglutinin

Abbreviations: PPA, porcine pancreatic $\alpha$-amylase; HPA, human pancreatic $\alpha$-amylase; $\alpha$-AI1, $\alpha$-amylase inhibitor from kidney bean (Phaseolus vulgaris) type 1; PHA, phytohemagglutinin; $i$, vertical axis intercept; $s$, slope; DP, degree of polymerization; $K_{1 i}, K_{2 i}, L_{1 i}$, and $L_{2 i}$, dissociation equilibrium constants; k3, rate constant; Enzyme, retaining $\alpha$ $(1 \rightarrow 4)$-glucan-4-glucanohydrolase (EC 3.2.1.1)

* Corresponding author. Fax: +33-4-91-28-84-40.

E-mail address: veronique.desseaux@univ.u-3mrs.fr (V. Desseaux).
(PHA), arcelin, and $\alpha$-amylase inhibitor ( $\alpha$-AI). These three proteins have quite different modes of action. PHA is a lectin that binds to the glycans of the intestinal mucosa of mammals and acts as a mitogen while arcelin, which is also a lectin-like protein, binds to the peritrophic membrane of insect larvae, thus preventing nutrient absorption. At last, $\alpha-$ AI (a truncated lectin) [1] inhibits the activity of certain mammalian and insect $\alpha$-amylases but not that of plant enzymes. Three different isoforms of $\alpha$-AI: $\alpha$-AI1 [2,3], $\alpha$ AI2 $[3,4]$ and $\alpha$-AI3 (or $\alpha$-AIL) [5] have been described. $\alpha$ $\mathrm{AI} 1$, a $43 \mathrm{kDa}$ dimeric glycoprotein $\left(\alpha_{2} \beta_{2}\right)[2,6]$ is homologous to PHA. Present in high amount in common bean seeds, it inhibits salivary and pancreatic $\alpha$-amylases of 
insects, specially bruchid larvae such as the cowpea and the azuki bean weevils, thus preventing starch digestion. $\alpha$-AI1, the main $\alpha$-AI isoform, exhibits the same overall jelly roll fold as PHA-L of the kidney bean (P. vulgaris) [7] but readily differs from the lectin [8] by the truncation on the back face of the protomer of two extended loops of 15 and 6 residues, respectively (Fig. 1) [9-11]. These loops are lacking in $\alpha$ -
AI2, which also corresponds to a truncated lectin. Arcelins Arce-1 [12] and Arce-5 [13] also differ from PHA-L by the single truncation of the shorter loop of six residues (Fig. 1). The inactive $\alpha$-AIL most probably corresponds to an evolutionary intermediate between PHA, arcelins and the active $\alpha$ amylase inhibitors [5]. Arcelins and $\alpha$-AIs also differ from PHA-L according to the degree of oligomerization (Fig. 1).
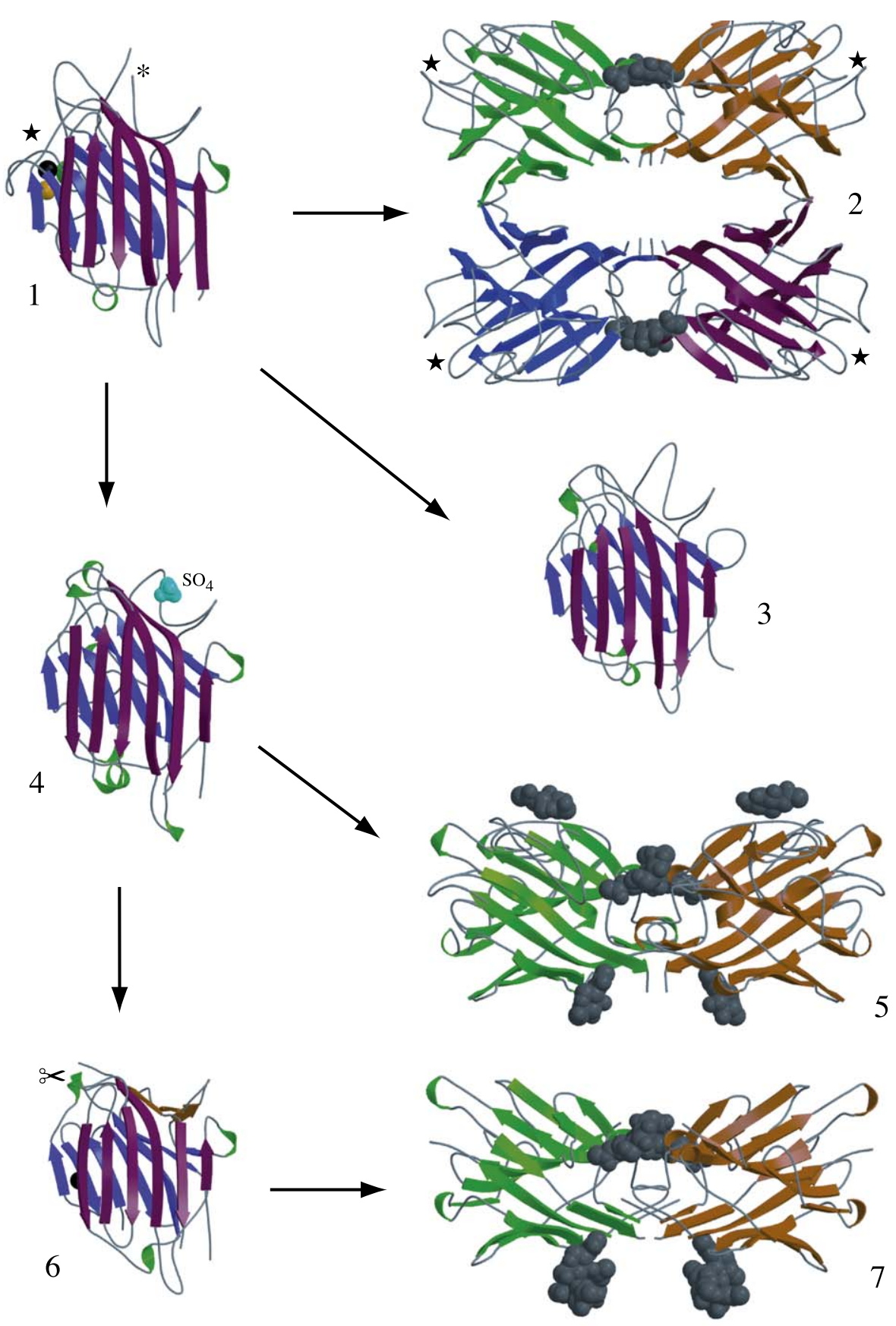

Fig. 1. Ribbon diagrams showing the relationship between the three-dimensional structures of the protomers of PHA-L (1) (RCSB PDB code 1FAT), Arce-5 (3) (RCSB PDB code 1IOA), Arce-1 (4) (RCSB PDB code 1AVB), and $\alpha$-AI1 (6) (RCSB PDB code 1DHK), the tetramer (dimer of dimer) of PHA-L (2), and the dimers of Arce-1 (5) and $\alpha$-AI1 (7). The bivalent cations are represented by black and grey balls. The fist GlcNAc $N$-linked to the Asn residues of the lectin-like dimers are in grey CPK. The carbohydrate-binding sites of PHA are indicated by stars $(\star)$, and the asterisk $(*)$ indicates the missing region in the threedimensional structure of the PHA protomer. $\&<$ indicates the proteolytic cleavage that generates the $\alpha$ - and $\beta$-chain of $\alpha$-AI1. All the protomers are represented by their flattened back face. Cartoons drawn with Molscript [9] and rendered with Bobscript [10] and Raster3D [11]. 
PHA-L is a tetramer built-up from the non-covalent association of two canonical 12-stranded $\beta$-sandwich dimers resulting from the non-covalent assembly of two protomers, through hydrophobic interactions between the upper edges of their back walls. Attempting to associate dimers of Arce-1 or $\alpha$-AI1 as in the PHA-L tetramer creates steric clashes susceptible to prevent the tetrameric assembly that could explain why native Arce- 1 and $\alpha$-AIl only exist as dimers. Moreover, the branched glycans linked to Asn ${ }^{68}$ of Arce-1 and to $\mathrm{Asn}^{65}$ and $\mathrm{Asn}^{140}$ of $\alpha$-AI1 residues, protrude in the solvent at the back face of the dimer and thus might prevent the formation of tetrameric structures by creating a steric conflict between two facing dimers.

The elimination of two loops on the front face of the lectin-like protomer appears as a structural requirement for the $\alpha$-amylase inhibitory activity of $\alpha$-AIs. The occurrence of one (Arce-1, Arce-5) or two extra-loops ( $\alpha$-AIL, PHA-L) prevents these proteins from entering the substrate cleft of the porcine pancreatic $\alpha$-amylase (PPA) (Fig. 2). In addition, a proteolytic processing occurring at $\mathrm{Asn}^{77}$ residue, which cleaves the polypeptide chain of $\alpha$-AI1 in two $\alpha$ - (residues $1-77$ ) and $\beta$-chain (residues 78-223) [14], is apparently necessary to transform an inactive precursor in a fully active inhibitor. As a result, some local conformational changes allows $\alpha$-AI1 to interact with the active cleft of $\alpha$-amylases.

$\alpha$-Amylases catalyse the hydrolysis of internal $\alpha-(1 \rightarrow 4)$ glucosidic linkages; these retaining glycosidases belong to the 13 family glycoside hydrolase [15]. They are widely distributed among Archaea, Bacteria and Eucarya [16,17]. The 496-amino acid sequence of PPA has been determined $[18,19]$ and the three-dimensional structure obtained at 0.2 $\mathrm{nm}$ resolution $[20,21]$. Following the $(\beta / \alpha)_{8}$ barrel (residues 1-405), PPA contains a $C$-terminal $\beta$-stranded domain (residues 406-496) with an $\alpha$-crystalline topology.

Preliminary kinetic results obtained with the $p$-nitrophenyl- $\alpha$-D-maltoside substrate analogue show that the binding of $\alpha$-AIl to PPA is a slow process [22]. This remarkable feature has been confirmed for PPA [2] and also found with human pancreatic $\alpha$-amylase (HPA) [23]; maximum inhibition is reached after $30 \mathrm{~min}$ in contact.

Acarbose is a pseudotetrasaccharidic inhibitor of $\alpha$ amylase that acts like a transition state analogue. The crystallography of the PPA-acarbose complex shows that acarbose binds to the active site $[7,24]$.

Kinetic studies have been carried out recently to determine the mechanism underlying the inhibitory effect of acarbose, using substrates of various lengths $[25,26]$. The inhibition is of the mixed noncompetitive type and either one or two molecules of acarbose bind(s) to PPA, whatever the substrate. These data led us to propose a general mechanism of PPA action involving two additional carbohydrate binding sites. The role of these two sugar-recognition sites and their link with the active site, possibly in the product processing, have not yet been elucidated.

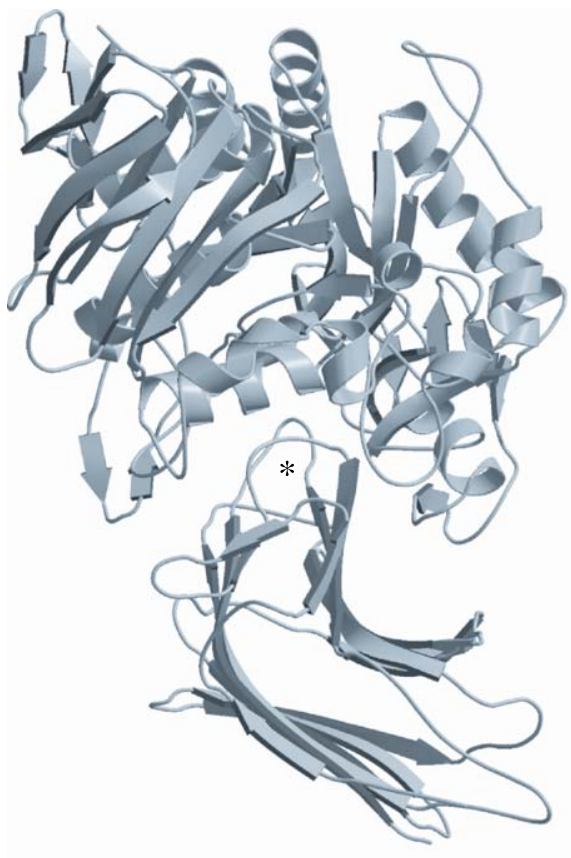

$\alpha-\mathrm{AI}-1$

\section{$\alpha$-amylase}

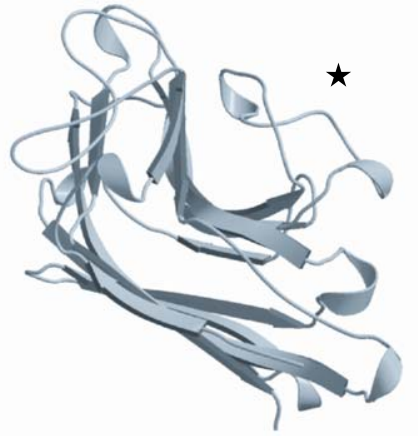

Arce-1

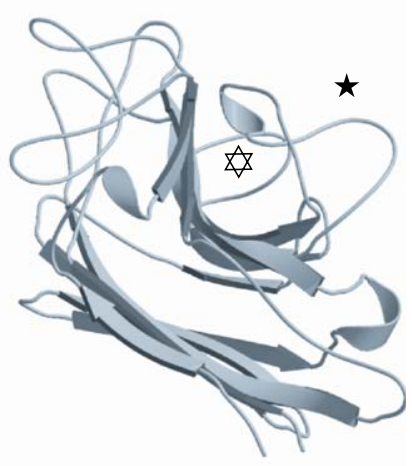

PHA-L

Fig. 2. Ribbon diagram showing the complex between $\alpha$-AI1 and the PPA (RCSB PDB code 1DHK). The Arce-1 (RCSB PDB code 1AVB) and PHA-L (RCSB PDB code 1FAT) protomers are represented in the same orientation as $\alpha$-AI1. The two hairpin loops anchoring $\alpha$-AI1 into the active cleft of PPA are indicated $(*)$. The two extra loops present in PHA-L ( $\star$ and $\star$ ) and the single extra loop occurring in Arce-1 $(\star)$ that prevent the binding to the active cleft of $\alpha$-amylase are indicated. Cartoons drawn with Molscript [9] and rendered with Bobscript [10] and Raster3D [11]. 
The crystal structure of the $\alpha$-AI1-PPA complex has been refined to $1.85 \AA$ resolution [7,27]. It shows that two hairpin loops of each monomer interact directly with the active site region of the enzyme molecule yielding an $\mathrm{E}_{2} \mathrm{I}$ complex (see this issue, Payan et al.).

In the present paper [28], the inhibitory effects of $\alpha$-AI1 on the PPA catalysed hydrolysis DP 4900-amylose (a long chain substrate) and maltopentaose (a short one) are described. The type of inhibition exerted by $\alpha$-AI1 is discussed. The proposed model accounts for the slow binding of the inhibitor to the enzyme and for crystallographical, biochemical and physicochemical data. The interactions of this lectin-like protein with PPA and the structural change leading to inhibition are further examined.

\section{Methods}

Kinetic experiments and X-ray structural analysis were performed as previously described [7,27]. Limited proteolysis of the $\alpha$ AI1 - PPA complex: samples in $50 \mathrm{mM}$ Tris/HCl buffer ( $\mathrm{pH}$ 7.4) containing $1 \mathrm{mM}$ sodium azide were treated with $2 \%$ subtilisin for $24 \mathrm{~h}$ at $30{ }^{\circ} \mathrm{C}$ and the digestion was stopped by adding $1 \mathrm{mM}$ di-isopropylfluoro-phosphate [29]. SDS-PAGE was performed by the method of Laemmli [30] using $12 \%$ acrylamide slab gels. Molecular weight determinations were made using molecular weight standards (low range kit Biorad). After electrophoresis, the gels were stained for protein with Coomassie brilliant blue R-250 or silver nitrate solution. For the N-terminal sequence analysis, gels were equilibrated for 10-20 min with transfer buffer $(50 \mathrm{mM}$ Tris/borate, $\mathrm{pH} 8$ ), then electro-blotted onto $0.1-\mu \mathrm{m}$-thick Immobilon TM-PSQ (Millipore Bedford, MA, USA) which were stained with Ponceau red solution.

\section{Results and discussion}

\section{1. $\alpha$-AI1 inhibition of PPA-catalysed amylose and} maltopentaose hydrolysis

As reported previously [2], no immediate inhibition was observed when substrate, PPA and $\alpha$-AI1 were mixed together. PPA was preincubated with $\alpha$-AI1 before adding the substrate to the reaction mixture. A prolonged preincubation period of $\alpha$-AI1 and PPA at $30{ }^{\circ} \mathrm{C}$ of $2 \mathrm{~h}$ for amylose and 30

A

\section{$[\alpha \mathrm{Al}]$}
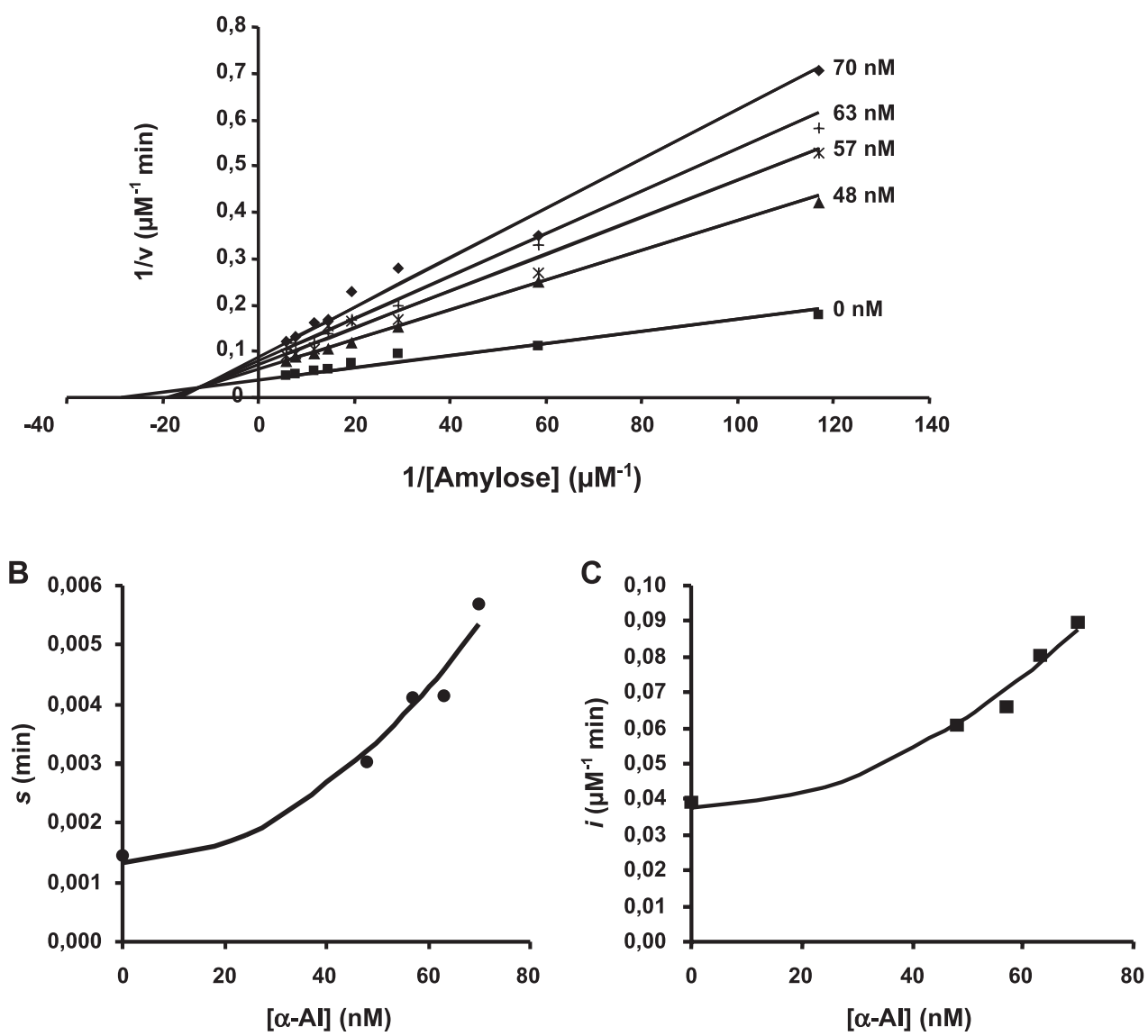

Fig. 3. Lineweaver-Burk plot using amylose as substrate. (A) Reciprocal plots obtained with variable amylose concentrations and at fixed concentration of the $\alpha$-AI1 inhibitor. The reciprocal plots are drawn from one set of data. (B,C) Secondary plots showing the dependence of the vertical axis intercept (i) and of the slope $(s)$ on the concentration of $\alpha$-AI1 inhibitor. 
min for maltopentaose was adopted. The incubation period ensures that complete equilibrium is reached between the enzyme, the inhibitor and the enzyme-inhibitor complex and allows us to postulate that the mechanism is at equilibrium. This assumption fits with experimental results: all the Lineweaver-Burk plots obtained were linear (Figs. 3A and 4A).

\subsubsection{Statistical analysis of kinetic data}

Whatever the type of inhibition involved (competitive, uncompetitive or noncompetitive) and assuming the rapid equilibrium hypothesis, the initial velocity $(v)$ fits the following general equation:

$v=\frac{C[\mathrm{~S}]}{A_{0}+B_{0}[\mathrm{~S}]+B_{1}[\mathrm{~S}][\mathrm{I}]+A_{1}[\mathrm{I}]}$

where $A_{0}, A_{1}, B_{0}, B_{1}$ and $C$ are parameters depending on equilibrium constants and $[\mathrm{S}]$ and $[\mathrm{I}]$ are the substrate and inhibitor concentrations, respectively.

This equation is valid when one molecule of $\mathrm{I}$ is involved. When the inhibition is noncompetitive, $A_{1}$ and $B_{1}$ are positive. If $B_{1}=0$, the inhibition is competitive, whereas if $A_{1}=0$, the inhibition is of the uncompetitive type.

When two molecules of inhibitor are involved in the binding process with either the enzyme and/or the enzymesubstrate complex, the denominator is a second-order polynomial expression with respect to [I]. In this case, the equation obtained is:

$v=\frac{C[\mathrm{~S}]}{A_{0}+B_{0}[\mathrm{~S}]+B_{1}[\mathrm{~S}][\mathrm{I}]+B_{2}[\mathrm{~S}][\mathrm{I}]^{2}+A_{1}[\mathrm{I}]+A_{2}[\mathrm{I}]^{2}}$

where $A_{0}, A_{1}, A_{2}, B_{0}, B_{1}, B_{2}$ and $C$ are parameters depending on equilibrium constants and [S] and [I] are the substrate and inhibitor concentrations, respectively.

This equation can be rewritten as [31]:

$\frac{[\mathrm{S}]}{v}=a_{0}+a_{1}[\mathrm{I}]+a_{2}[\mathrm{I}]^{2}+[\mathrm{S}]\left(b_{0}+b_{1}[\mathrm{I}]+b_{2}[\mathrm{I}]^{2}\right)$

where $a_{0}, a_{1}, a_{2}, b_{0}, b_{1}$ and $b_{2}$ are coefficients depending on the equilibrium constants.

A

$[\alpha$ Al]
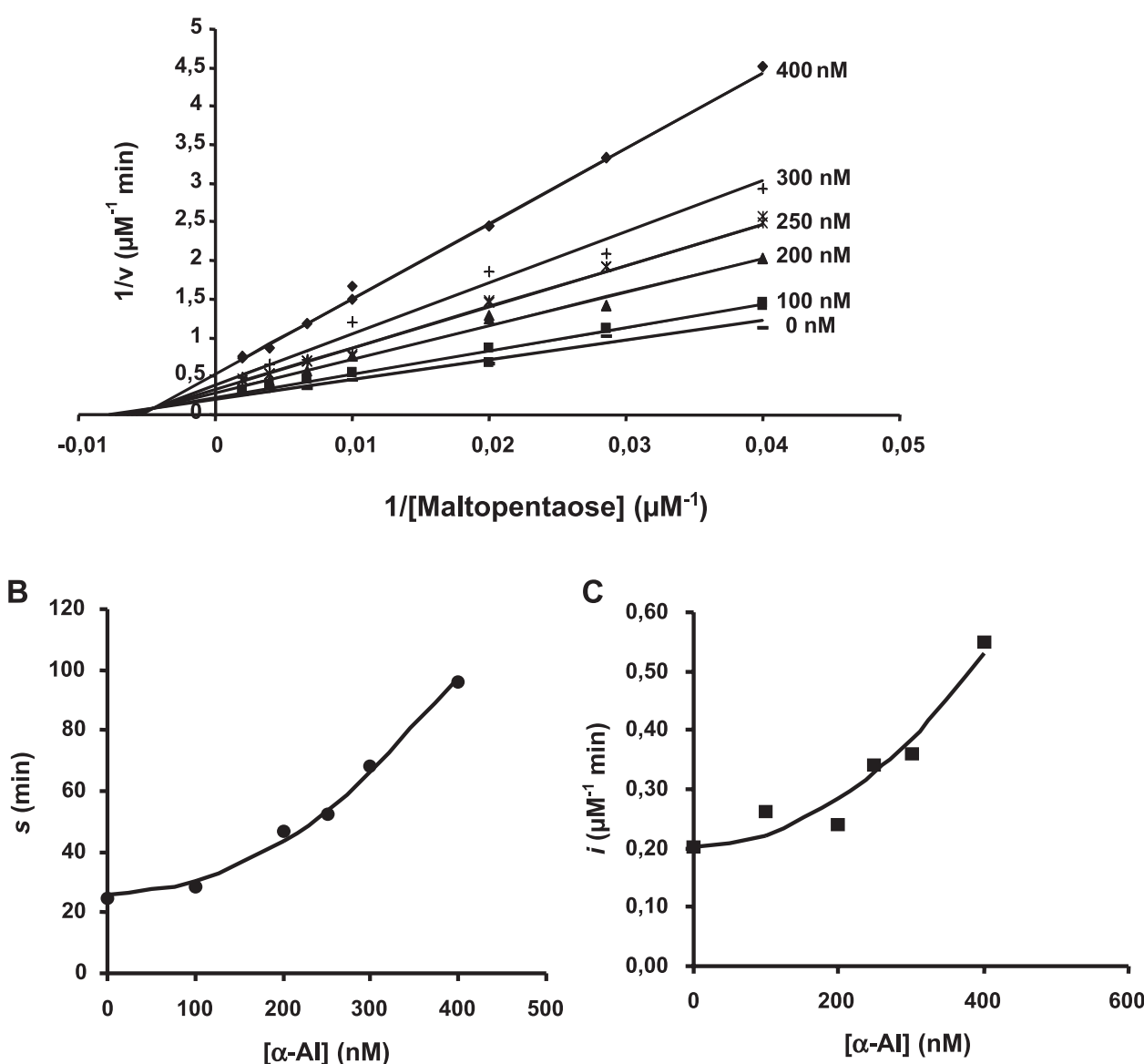

Fig. 4. Lineweaver-Burk plot using maltopentaose as substrate. Reciprocal plots obtained with variable maltopentaose concentrations and at fixed concentration of the $\alpha$-AI1 inhibitor. The reciprocal plots are drawn from one set of data. (B,C) Secondary plots showing the dependence of the vertical axis intercept $(i)$ and of the slope $(s)$ on the concentration of $\alpha$-AI1 inhibitor. 
It should be pointed out that the $[\mathrm{S}] / v$ equation (Eq. (3)) is linear with respect to [S] at fixed I concentration at rapid equilibrium; in the steady state, the initial velocity calculated by the King and Altman method [32] also gives a linear $[\mathrm{S}] / v$ versus $[\mathrm{S}]$ equation except with a model of random type [25].

The experimental kinetic data were adjusted to Eq. (3) by performing a polynomial regression analysis, which allows to test whether or not coefficients $a_{1}, a_{2}, b_{0}, b_{1}$ and $b_{2}$ are significant. Note that coefficients $a_{1}, a_{2}$ and $b_{1}, b_{2}$ correspond to the presence of $\mathrm{EI}, \mathrm{EI}_{2}$ and $\mathrm{ESI}, \mathrm{ESI}_{2}$ enzyme species in the reaction mixture. The results of this analysis are summarized in Table 1. With both substrates (amylose and maltopentaose), only coefficients $b_{0}, b_{2}$ and $a_{2}$ differed significantly from zero, while coefficients $b_{1}$ and $a_{1}$ did not.

$\mathrm{EI}_{2}$ and $\mathrm{ESI}_{2}$ were therefore present in the reaction medium in significant concentrations, while EI and ESI were probably present in very low concentrations. This agrees with the binding of two inhibitor molecules to both the free enzyme and the enzyme-substrate complex.

\subsubsection{Lineweaver-Burk plots and replots}

Eq. (3) can be rewritten as follows:

$\frac{1}{v}=\left(a_{0}+a_{1}[\mathrm{I}]+a_{2}[\mathrm{I}]^{2}\right) \frac{1}{[\mathrm{~S}]}+b_{0}+b_{1}[\mathrm{I}]+b_{2}[\mathrm{I}]^{2}$

Lineweaver-Burk plots and the corresponding secondary plots fit this equation (Figs. 3 and 4). The primary plots of the inhibition of amylose (Fig. 3A) and maltopentaose (Fig. 4A) hydrolysis were similar. Straight lines were obtained with each substrate. Both the slope $s$ and the vertical axis intercept $i$ increased with increasing of the $\alpha$-AI1 inhibitor concentrations [I]. The analysis of these results by the Cleland method [33] accounts for noncompetitive inhibition. In addition, in the primary plots, the intercepts were in the second quadrant. The inhibition was therefore of the mixed noncompetitive kind (Table 2).

As in competitive inhibition, the effects of the $\alpha-\mathrm{AI} 1$ concentration, $[\mathrm{I}]$, on the slope indicate that I binds to the free enzyme E, while the effects of the $\alpha$-AIl concentration on

Table 1

Polynomial regression analysis of the kinetic data

\begin{tabular}{llll}
\hline Substrate & Coefficient & $d f$ & $\mathrm{P}>F$ \\
\hline Amylose & $b_{0}$ & 34 & 0.0001 \\
& $b_{1}$ & 34 & 0.4735 \\
& $b_{2}$ & 34 & 0.0104 \\
$a_{1}$ & 34 & 0.4779 \\
Maltopentaose & $a_{2}$ & 34 & 0.0164 \\
& $b_{0}$ & 78 & 0.0001 \\
& $b_{1}$ & 78 & 0.0885 \\
& $b_{2}$ & 78 & 0.0001 \\
& $a_{1}$ & 78 & 0.4790 \\
$a_{2}$ & 78 & 0.0001 \\
\hline
\end{tabular}

The coefficients are from Eq. (3). $d f$, degree of freedom; $F$, Fisher statistical significance (type III) with the probability $P$ of finding an $F$ value greater than the calculated ones.
Table 2

The kinetics parameters and inhibition by $\alpha$-AI1 acting on amylose and maltopentaose

\begin{tabular}{llllrl}
\hline Substrate & Inhibitor & $\begin{array}{l}10^{3} k_{\text {cat }} \\
\left(\mathrm{s}^{-1}\right)\end{array}$ & $\begin{array}{l}k_{\text {cat }} / K_{\mathrm{m}} \\
\left(\mathrm{s}^{-1}\right. \\
\left.\mathrm{mg}^{-1} 1\right)\end{array}$ & $\begin{array}{l}\text { Inhibition } \\
(\%)\end{array}$ & $\begin{array}{l}\text { Inhibition } \\
\text { type }\end{array}$ \\
\hline Amylose & no & 1.20 & 62 & 0 & \\
& $\alpha-\mathrm{AI} 1$ & 0.69 & 16 & 43 & mixed \\
Maltopentaose & no & 0.081 & 0.83 & 0 & noncompetitive \\
& $\alpha$-AI1 & 0.033 & 0.36 & 59 & \\
\hline
\end{tabular}

the vertical axis intercept show that I binds to the enzymesubstrate complex, ES, as in uncompetitive inhibition.

The secondary Lineweaver-Burk plots of the amylose (Fig. 3B,C) and maltopentaose hydrolysis (Fig. 4B,C) were also similar: plotting both the slope $s$ and the vertical axis intercept $i$ against the inhibitor concentration produced parabolic lines. The respective second-order equations of these lines with respect to inhibitor concentration [I] illustrate the results of the above statistical analysis: two I molecules bind to both the free enzyme and the enzymesubstrate complex. In contrast, as shown by X-ray analysis of the PPA- $\alpha$ AI1 complex, one I molecule binds to the active site while the other one occupies a secondary binding site (as in $\mathrm{EI}_{2}$ and $\mathrm{ESI}_{2}$ ).

\subsubsection{Inhibition kinetic models}

The inhibitory effects on PPA-catalysed amylose and maltopentaose hydrolysis were previously reported using pseudotetrasaccharide acarbose as the inhibitor $[25,26]$. Lineweaver-Burk plots were analysed using the Cleland method [33]: the effects of the acarbose inhibitor concentration on the slope and on the vertical axis intercept of the primary plots were studied. Inhibition was found to be of the noncompetitive type involving either one or two molecules of inhibitor, whether the substrate was maltopentaose or amylose. Two models (1 and 2) were proposed, involving abortive complexes.
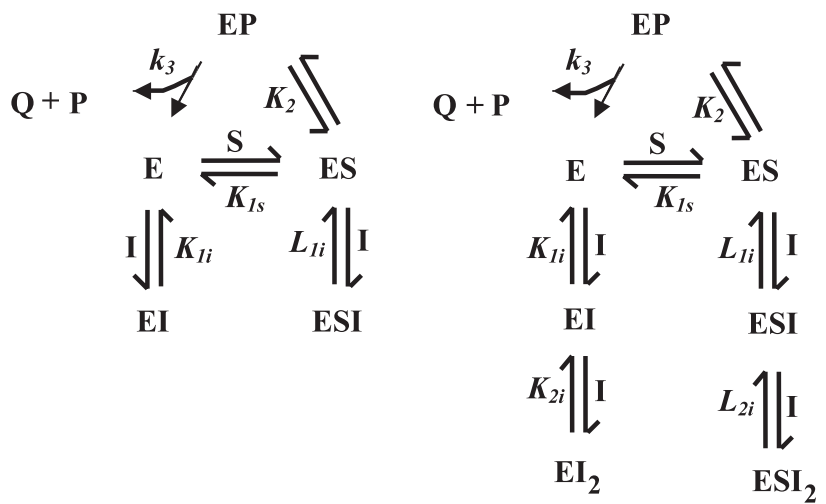

Model 1

$(\mathrm{S}=$ maltopentaose $)$
Model 2

( $\mathrm{S}=$ amylose $)$
(P and Q are products obtained from substrate hydrolysis) 
Do these models fit the data obtained in the case of $\alpha$-AI1 inhibition? Model 1 has to be ruled out because only one I molecule binds to both the enzyme E and the enzymesubstrate complex ES. Model 2 agrees with the statistical analysis of the $\alpha$-AIl inhibition, as two I molecules bind to both the free enzyme E and the enzyme complex ES as complexes $\mathrm{EI}_{2}$ and $\mathrm{ESI}_{2}$, respectively. However, this model predicts that inhibition will occur without any preincubation of the inhibitor with the enzyme. When the enzyme is actually added to the reaction mixture containing the substrate (amylose/maltopentaose) and the $\alpha$-AI1 inhibitor, no inhibition occurs at first: the inhibitor concentration has no effect on the slope or on the vertical axis intercept of the primary plots. Inhibition is observed only when the enzyme and the inhibitor have been initially preincubated for at least $10 \mathrm{~min}$ before addition of the substrate. This suggests that neither EI nor the ESI complex is present in detectable amounts at the beginning of the reaction and consequently that both reactions:

$$
\mathrm{E}+\mathrm{I} \stackrel{k_{1}}{\underset{k-1}{\rightleftharpoons}} \mathrm{EI} \text { and } \mathrm{ES}+\mathrm{I} \stackrel{l_{1}}{\underset{l_{-1}}{\longrightarrow}} \mathrm{ESI}
$$

are slow reactions with low rate constants, $k_{1}$ and $l_{1}$, respectively. It was previously reported that EI formation is a slow process $[2,22]$. When the inhibitor is not preincubated with the enzyme, because the formation of EI and ESI is slow, these complexes are not formed when the initial velocity is measured, therefore no immediate inhibition might be observed. Therefore, Model 2' may be valid if it is assumed that the I binding at $\mathrm{E}$ or $\mathrm{ES}$ is a slow reaction.

Is this model still valid when E and I are preincubated?
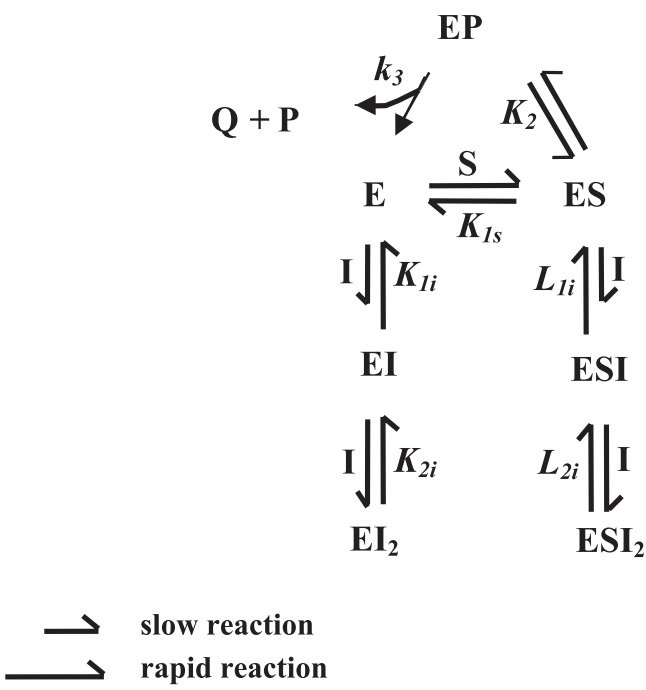

Model 2'

When E and I are preincubated, the complex EI is already formed when the substrate is added to the medium, and the inhibitory effect on the slope is observed as predicted by the
Model $2^{\prime}$. Also, upon addition of the substrate to the reaction medium, the complex ES is immediately formed and the hydrolysis begins immediately, while the binding of I to ES is slow and the amount of ESI complex produced is likely to be very small and have no effect on the vertical axis intercept: this contradicts the data obtained here by measuring the initial velocity as the effect on the vertical axis intercept is observed. Therefore, Model 2' cannot be retained.

To account for the immediate effect observed on the vertical axis intercept upon adding the substrate to the preincubated reaction medium containing $\mathrm{E}$ and $\mathrm{I}$, one has to assume that the ESI complex is not produced by binding of I to the ES complex but that it is obtained via a different pathway, in which the inhibitor slowly binds to the enzyme before the rapid substrate binding to the EI complex occurs, giving ESI:

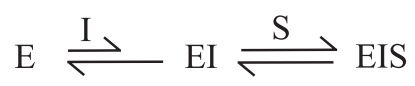

In line with the last hypothesis, we propose Model 3.

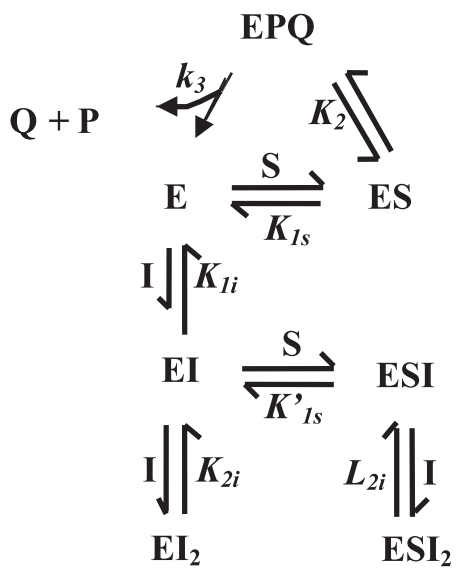

Model 3

At equilibrium, the initial velocity corresponding to this model is given by Eq. (5):

$v=\frac{V[\mathrm{~S}]}{K_{\mathrm{m}}\left(1+\frac{[\mathrm{I}]}{K_{1 i}}+\frac{[\mathrm{I}]^{2}}{K_{1 i} K_{2 i}}\right)+[\mathrm{S}]\left(1+\frac{K_{\mathrm{m}}}{K_{1 s}^{\prime}} \frac{[\mathrm{I}]}{K_{1 i}}+\frac{K_{\mathrm{m}}}{K_{1 s}^{\prime}} \frac{[\mathrm{I}]^{2}}{K_{1 i} L_{2 i}}\right)}$

where

$V=\frac{k_{3}}{1+K_{2}}[E]_{0}$ and $K_{\mathrm{m}}=\frac{K_{1 s} K_{2}}{1+K_{2}}$

Similar equation is obtained at steady state. This equation corresponds to the parametric Eq. (2). 
The inhibition mechanism can actually takes into account the X-ray diffraction data of the inhibitor-PPA complex. In the crystal structure, the inhibitor I binds to two E molecules forming a single $E_{2} I$ complex and occupies the active sites [7]. Also, the results of studies using gel-filtration and lightscattering techniques applied to the analysis of the $\mathrm{E}+\mathrm{I}$ preincubated mixture, led to the characterization of two complexes, $\mathrm{E}_{2} \mathrm{I}$ and $\mathrm{EI}[6]$. This discrepancy with the EI and $\mathrm{EI}_{2}$ complexes deduced from the inhibition kinetics can be explained by the following: when $\mathrm{E}$ and I are mixed, the first elementary reaction corresponds to the EI complex formation. Then, the complex formation depends on the $[\mathrm{I}] /$ [E] ratio used, and two pathways are possible:

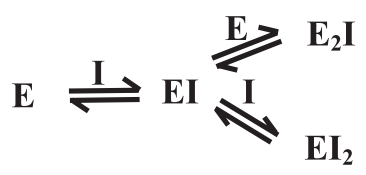

At a ratio of 1 only $\mathrm{E}_{2} \mathrm{I}$ is observed (crystallographic data [7]) since in the elementary reaction, the equilibrium is displaced towards the formation of $\mathrm{E}_{2} \mathrm{I}$. At a ratio of 2 the $\mathrm{E}_{2} \mathrm{I}$ and the EI complexes are observed (biophysical data [6]) since the elementary reaction is equilibrated. In the present kinetic experiments, the ratio $[\mathrm{I}] /[\mathrm{E}]$ used is much higher (160-233 when amylose was used as the substrate and 100-400 with maltopentaose), the EI and the $\mathrm{EI}_{2}$ complexes are present (see scheme above). Moreover, because of the substrate, the ESI and $\mathrm{ESI}_{2}$ can also be obtained.

The following general model (Model 4) takes both the kinetic results and the crystallographic and gel filtration data into account. In particular, this model explains how two different complexes $\left(\mathrm{EI}_{2}\right.$ and $\left.\mathrm{E}_{2} \mathrm{I}\right)$ can be obtained from the same initial complex EI. Therefore, in crystallographic and biophysical studies, $\mathrm{E}_{2} \mathrm{I}$ or EI and $\mathrm{E}_{2} \mathrm{I}$ complexes, respectively, are obtained while $\mathrm{EI}$ and $\mathrm{EI}_{2}$ complexes occur in kinetic experiments.

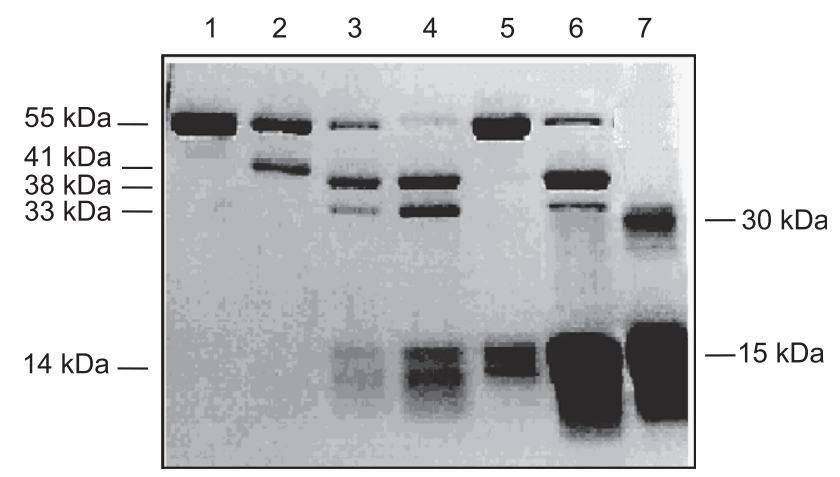

Fig. 5. SDS-PAGE electrophoretic analysis. Protein samples $1.5 \mu \mathrm{g}$ except lane $7(5 \mu \mathrm{g})$, were run on $12 \%$ and stained with Coomassie blue. PPA (lane 1), PPA-subtilisin $2 \%$ (lane 2), $\alpha$-AIl (lane 7) or $\alpha$-AI-subtilisin $2 \%, \alpha$ AI1 - PPA at an $\alpha$-AI1 - PPA ratio of 1 (lane 5), $\alpha$-AI1-PPA-subtilisin $2 \%$ with an $\alpha$-AI1-PPA ratio of 0.4 (lane 3), 1 (lane 4) and 10 (lane 6). The molecular masses are indicated on both sides.

\subsection{Limited proteolysis of PPA in the $\alpha-A I 1-P P A$ complex}

$\alpha$-AI1, PPA and the preincubated $\alpha$-AI1-PPA mixture for $15 \mathrm{~min}, 1,2$ or $3 \mathrm{~h}$ at $37{ }^{\circ} \mathrm{C}$ in the buffer $\mathrm{pH} 7.4$ at the indicated $\alpha$-AI1-PPA ratios (see legend of Fig. 5), were treated with subtilisin. Without preincubation, the PPA with $\alpha$-AI1 is not proteolysed. $\alpha$-AI1 and $\alpha$-AI1 subtilisin-treated samples give similar patterns: two groups of bands, one at about $30 \mathrm{kDa}$ and the other at about $15 \mathrm{kDa}$ (Fig. 5, lane 7). The protein fraction of $M_{\mathrm{r}} 30 \mathrm{kDa}$ probably corresponds to the unprocessed $\alpha \mathrm{AI} 1$ proportion, as previously suggested by Moreno and Chrispeels [34]. That is confirmed by the Nterminal sequences which show subunits $(\alpha+\beta)$ against one subunit $(\beta)$. This fraction will be partially cleaved under denaturing conditions SDS-PAGE. The $15 \mathrm{kDa}$ region is rather broad because the N-terminal sequences contain two kinds of glycopolypeptide subunits, $\alpha, \varepsilon(\alpha+\beta)$ and $\beta$ in variable proportions. This shows that under these conditions $\alpha$-AI1 is not susceptible to subtilisin attack (lane 7). PPA control (lane 1) migrates as a single band at $55 \mathrm{kDa}$. After subtilisin digestion, about half of the PPA is cleaved into 41 and $14 \mathrm{kDa}$ fragments (lane 2) where about $50 \%$ of the activity was gone [29].

When the $\alpha$-AI1-PPA complex was digested with subtilisin at 0.4 (lane 3), 1 (lane 4) and 10 (lane 6) $\alpha$-AI1-PPA ratio, almost complete hydrolysis of PPA occurred at this stage, most activity was lost and three bands of 38,33 and $14 \mathrm{kDa}$ were obtained. Therefore, at high I inhibitor concentration ( $\alpha$-AI1-PPA $\geq 1)$, the $41 \mathrm{kDa}$ fragment is not present and further digestion of PPA is obtained when in complex with $\alpha$-AI1. The N-terminal sequence of both the 38 and $33 \mathrm{kDa}$ fragments was: $\mathrm{Thr}^{6}, \mathrm{Gln}^{7}, \mathrm{Ser}^{8}, \mathrm{Gly}^{9}, \mathrm{Arg}^{10}$, $\mathrm{Thr}^{11}$. These fragments therefore both originate from the 41 $\mathrm{kDa}$ component. Five residues were removed from the Nterminal pyroGlu end. These fragments were also shorter at the C-terminus than the $41 \mathrm{kDa}$ compound. These results show that PPA is much more sensitive to subtilisin attack in the inhibitor-enzyme complex, which suggests that the PPA

Model 4 
conformation is changed. This is in agreement with the change in the three-dimensional structure, as detected by Fourier difference analysis [7].

\subsection{Structure of the $\alpha-A I 1$ and of the $\alpha-A I 1-P P A$ complex and comparison with the kinetic analysis}

Some of our experimental data are in full agreement with the already described X-ray $\alpha$-AI1 -PPA complex. $\alpha$-AI1 complexed to PPA showed that a network of $15 \mathrm{H}$-bonds allows the anchoring of the inhibitor into the active cleft of $\alpha$-amylase [7]. The major interaction occurs inside the Vshaped active site of PPA with two hairpin loops L1 (residues 29-46) and L2 (residues 171-189) protruding on the upper side of the front face of the $\alpha$-AI1 protomer (Fig. 2). $\alpha$-AI1 was similarly shown to interact with TMA, the Tenebrio molitor $\alpha$-amylase [35]. Upon binding of $\alpha$ AI1 to PPA, some structural changes of the enzyme were shown to occur and, especially, the flexible loop 304-310 moves towards the solvent, thus allowing the $\alpha$-AI1 inhibitor to insert the two protruding loops L1 and L2 into the active cleft of PPA [7]. This conformational change provokes a readjustment of the surrounding regions of the enzyme and other loops of the PPA molecule are similarly displaced in response to the inhibitor insertion. These structural changes are not immediate, which readily explains why PPA has to be preincubated with $\alpha$-AIl before the substrate is added in order to get inhibition in kinetic experiments. In addition, they probably account for the enhanced susceptibility to subtilisin of the enzyme complexed to the inhibitor, e.g. by allowing a better accessibility of both the $\mathrm{N}$ - and C-terminal end of PPA to the proteolytic enzyme.

How to reconcile the X-ray observations with the kinetics model? The crystal structure of the $\alpha$-AI1-PPA complex revealed that the dimeric $\alpha$-AI1 inhibitor interacted with two enzyme molecules [7] as an $\mathrm{E}_{2} \mathrm{I}$ complex. The binding of each $\alpha$-AI1 monomer at the V-shaped catalytic cleft of PPA was unambiguously demonstrated. Moreover, each $\alpha$-AI1 monomer completely prevents the access of the enzyme cavity to other ligands, whatever their size. Accordingly, the occurrence on the PPA molecule of another protein bindingsite, distinct from the main catalytic cleft, has to be postulated in order to account for the formation of the $\mathrm{EI}_{2}$ and $\mathrm{EI}_{2} \mathrm{~S}$ complexes. At present, the $\alpha$-AI1-PPA complex offers no structural evidence for the occurrence of such another protein binding-site on the surface of PPA. However, we have to keep in mind that the X-ray solved $\alpha$-AI1 PPA complex was obtained at an $[\mathrm{I}] /[\mathrm{E}]$ ratio very different from that used in the kinetic measurements, which presumably accounts for such a discrepancy. Using a much higher [I]/[E] ratio apparently leads to different complexes like $\mathrm{EI}_{2}$ or $\mathrm{EI}_{2} \mathrm{~S}$ for which no three-dimensional structure is yet available.

Comparisons between the $\alpha$-AI1 inhibitory mechanism and that previously proposed for acarbose help to under- stand the respective processes. The main difference between the acarbose and the $\alpha$-AI1 inhibition schemes is that the ternary complex ESI is obtained via two different pathways. In the $\alpha$-AI1 scheme (Model 4), one molecule of I binds slowly at the E active site to give EI which then accepts one $\mathrm{S}$ molecule at an additional site to give $\mathrm{E}-\mathrm{S}-\alpha$-AIl (ESI). Conversely, in the acarbose scheme (Model 2), one molecule of $\mathrm{S}$ is bound at the $\mathrm{E}$ active site to give the ES binary complex, then one molecule of I can bind to this complex to give the $\mathrm{E}-\mathrm{S}$-acarbose (ESI) ternary complex. It should be pointed out that, in the acarbose scheme, $\mathrm{S}$ occupies the $\mathrm{E}$ active site in the ESI complex, while acarbose is bound at an additional carbohydrate-binding site, whereas, in the $\alpha$-AI1 scheme, I occupies the E active site while $\mathrm{S}$ is bound at an additional protein binding site. These differences in the mechanism of PPA inhibition probably depend on the chemical type and the size of the inhibitor molecule. In fact, acarbose has no effect on the sensitivity of PPA to subtilisin in the PPA-acarbose complex (results not shown), whereas in the $\alpha$-AI1-PPA complex, higher sensitivity of PPA to subtilisin attack is observed. Such behaviour may originate from conformational changes. The binding of acarbose to PPA only results in a very small and discrete conformational change [24], whereas the binding of $\alpha$-AI1 to PPA results in very large conformational changes [7] and the subtilisin sensitivity to PPA is enhanced [28].

In conclusion, firstly, the preincubation of $\alpha$-AI1 and PPA to get inhibition (EI complex) is consistent with the entry of two hairpin loops into the PPA active site $\left(\mathrm{E}_{2} \mathrm{I}\right.$ in the crystal at high [E] concentration). Secondly, inhibition kinetic studies indicate that under our conditions, a carbohydrate binding site (EIS) and a protein binding site $\left(\mathrm{EI}_{2}\right.$ or $\mathrm{EI}_{2} \mathrm{~S}$ ) participate in the inhibitory mechanism. This second binding site is functional only when I is present in the active site.

\section{Acknowledgements}

We are very grateful to Mr. C. Villard for excellent technical assistance and to Mr. J. Bonicel (CNRS Marseille) for peptide sequencing. This paper is dedicated to the late Prof. E. Prodanov (Montevideo, Uruguay). This work had financial support from the CEE-grant CI1-CT94-0034 and from ECOS Action no. U93E04.

\section{References}

[1] P. Rougé, A. Barre, H. Causse, C. Chatelain, G. Porthé, Arcelin and $\alpha$-amylase inhibitor from the seed of common bean (Phaseolus vulgaris) are truncated lectins, Biochem. Syst. Ecol. 21 (1993) 695-703.

[2] V. Le Berre-Anton, C. Bompard-Gilles, F. Payan, P. Rougé, Characterization and functional properties of the $\alpha$-amylase inhibitor $(\alpha-\mathrm{AI})$ from kidney bean (Phaseolus vulgaris) seeds, Biochim. Biophys. Acta 1343 (1997) 31-40.

[3] L.M. Hoffman, D.D. Donaldson, Characterization of two Phaseolus 
vulgaris phytohaemagglutinin genes closely linked on the chromosome, EMBO J. 4 (1985) 883-889.

[4] K. Suzuki, M. Ishimoto, K. Kitamura, cDNA sequence and deduced primary structure of an $\alpha$-amylase inhibitor from a bruchid-resistant wild common bean, Biochim. Biophys. Acta 1206 (1994) 289-291.

[5] F. Finardi-Filho, E. Mirkov, M.J. Chrispeels, A putative precursor protein in the evolution of the bean $\alpha$-amylase inhibitor, Phytochemistry 43 (1996) 57-62.

[6] K. Kasahara, K. Hayashi, T. Arakawa, J.S. Philo, J. Wen, S. Hara, H. Yamaguchi, Complete sequence, subunit structure, and complexes with pancreatic alpha-amylase of an alpha-amylase inhibitor from Phaseolus vulgaris white kidney beans, J. Biochem. 120 (1996) $177-183$.

[7] C. Bompard-Gilles, P. Rousseau, P. Rougé, F. Payan, Substrate mimicry in the active center of a mammalian $\alpha$-amylase: structural analysis of an enzyme-inhibitor complex, Structure 4 (1996) 1441-1452.

[8] T.W. Hamelryck, M.H. Dao-Thi, F. Poortmans, M.J. Chrispeels, L. Wyns, R. Loris, The crystallographic structure of phytohemagglutinin-L, J. Biol. Chem. 271 (1996) 20479-20485.

[9] P.J. Kraulis, Molscript: a program to produce both detailed and schematic plots of protein structures, J. Appl. Crystallogr. 24 (1991) 946-950

[10] R.M. Esnouf, An extensively modified version of Molscript that includes greatly enhanced coloring capabilities, J. Mol. Graph. 15 (1997) $132-134$.

[11] E.A. Merritt, D.J. Bacon, Raster 3D photorealistic molecular graphics, Methods Enzymol. 277 (1997) 505-524.

[12] L. Mourey, J.D. Pédelacq, C. Birck, C. Fabre, P. Rougé, J.P. Samama, Crystal structure of the arcelin-1 dimer from Phaseolus vulgaris at 1.9 Åresolution, J. Biol. Chem. 273 (1998) 12914-12922.

[13] T.W. Hamelryck, F. Poortmans, A. Goossens, G. Angenon, M. Van Montagu, L. Wyns, R. Loris, Crystal structure of arcelin-5, a lectinlike defense protein from Phaseolus vulgaris, J. Biol. Chem. 271 (1996) 32796-32802.

[14] J.J. Pueyo, D.C. Hunt, M.J. Chrispeels, Activation of bean (Phaseolus vulgaris) $\alpha$-amylase inhibitor requires proteolytic processing of the proprotein, Plant Physiol. 101 (1993) 1341-1348.

[15] J.D. McCarter, S.G. Withers, Mechanisms of enzymatic glycoside hydrolysis, Curr. Opin. Struct. Biol. 4 (1994) 885-892.

[16] H.M. Jespersen, E.A. MacGregor, M.R. Sierks, B. Svensson, Comparison of the domain-level organization of starch hydrolases and related enzymes, Biochem. J. 280 (1991) 51-55.

[17] S. Janecek, Sequence similarities and evolutionary relationships of microbial, plant and animal alpha-amylases, Eur. J. Biochem. 224 (1994) 519-524.

[18] L. Pasero, Y. Mazzei-Pierron, B. Abadie, Y. Chicheportiche, G. Marchis-Mouren, Complete amino acid sequence and location of the 5 disulfide bridges in porcine pancreatic $\alpha$-amylase, Biochim. Biophys. Acta 869 (1986) 147-157.

[19] S. Darnis, N. Juge, X.J. Guo, G. Marchis-Mouren, A. Puigserver, J.C. Chaix, Molecular cloning and primary structure analysis of porcine pancreatic $\alpha$-amylase, Biochim. Biophys. Acta 35850 (1999) 1-9.

[20] G. Buisson, E. Duée, R. Haser, F. Payan, Three-dimensional structure of porcine pancreatic $\alpha$-amylase at $2.9 \AA$ resolution, role of calcium in structure and activity, EMBO J. 6 (1987) 3909-3916.
[21] M. Qian, R. Haser, F. Payan, Structure and molecular model refinement of pig pancreatic $\alpha$-amylase at $2.1 \AA$ resolution, J. Mol. Biol. 231 (1993) 785-799.

[22] E.R. Wilcox, J.R. Whitaker, Some aspects of mechanism of red kidney bean $\alpha$-amylase inhibitor and $\alpha$-amylase, Biochemistry 23 (1984) 1783-1791.

[23] V. Le Berre-Anton, V. Nahoum, M. Santimone, A. Barre, G. Marchis-Mouren, F. Payan, P. Rougé, Interaction of the bean (Phaaseolus vulgaris) $\alpha$-amylase inhibitors with human $\alpha$-amylases: structural and functional aspects, in: A.J.M. Jansman, G.D. Hill, A.F.B. Van Der Poel (Eds.), Recent Advances of Research on Antinutritional Factors in Legume seeds and Rapeseed, vol. 93. Wageningen Press, 1998, pp. 131-135.

[24] M. Qian, R. Haser, G. Buisson, E. Duee, F. Payan, The active center of mammalian $\alpha$-amylase. Structure of the complex of a pancreatic $\alpha$ amylase with a carbohydrate inhibitor refined to $2.2-\AA$ resolution, Biochemistry 24 (1994) 6284-6294.

[25] M. Al Kazaz, V. Desseaux, G. Marchis-Mouren, F. Payan, E. Forest, M. Santimone, The mechanism of porcine pancreatic $\alpha$-amylase. Kinetic evidence for two additional carbohydrate-binding sites, Eur. J. Biochem. 241 (1996) 787-796.

[26] M. Al Kazaz, V. Desseaux, G. Marchis-Mouren, E. Prodanov, M. Santimone, The mechanism of porcine pancreatic $\alpha$-amylase. Inhibition of maltopentaose hydrolysis by acarbose, maltose and maltotriose, Eur. J. Biochem. 252 (1998) 100-107.

[27] C. Gilles, P. Rousseau, P. Rougé, F. Payan, Crystallization and preliminary X-ray analysis of pig pancreatic $\alpha$-amylase in complex with a bean lectin-like inhibitor, Acta Crystallogr., D 52 (1996) 581-582.

[28] R. Koukiekolo, V. Le Berre-Anton, V. Desseaux, Y. Morreau, P. Rougé, G. Marchis-Mouren, M. Santimone, Mechanism of porcine pancreatic $\alpha$-amylase. Inhibition of amylose and maltopentaose hydrolysis by kidney bean (Phaseolus vulgaris) inhibitor and comparison with that by acarbose, Eur. J. Biochem. 265 (1999) 20-26.

[29] V. Desseaux, F. Payan, E.H. Ajandouz, B. Svensson, R. Haser, G. Marchis-Mouren, Effect of limited proteolysis in 8th loop of the barrel and of antibodies on porcine pancreas amylase activity, Biochim. Biophys. Acta 1080 (1991) 237-244.

[30] U.K. Laemmli, Cleavage of structural proteins during the assembly of the head of bacteriophage T4, Nature 227 (1970) 680-685.

[31] A. Cornish-Bowden, Analysis of Enzyme Kinetic Data, Oxford Univ. Press, Oxford, 1995.

[32] E.L. King, C. Altman, A schematic method of deriving the rate laws for enzyme catalyzed reactions, J. Phys. Chem. 60 (1956) 1375-1378.

[33] W.W. Cleland, The kinetics of enzyme catalyses reactions with two or more substrates or products, Biochim. Biophys. Acta 67 (1963) 173-196.

[34] J. Moreno, M.J. Chrispeels, A lectin gene encodes the $\alpha$-amylase inhibits of the common bean, Proc. Natl. Acad. Sci. U. S. A. 86 (1989) 7885-7889.

[35] V. Nahoum, F. Farisei, V. Le Berre-Anton, M.P. Egloff, P. Rougé, E. Poerio, F. Payan, A plant seed inhibitor of two classes of $\alpha$-amylases. $\mathrm{X}$-ray analysis of Tenebrio molitor larvae $\alpha$-amylase in complex with the bean Phaseolus vulgaris inhibitor, Acta Crystallogr., D 55 (1999) $360-362$. 